Ciência Florestal, Santa Maria, v. 21, n. 2, p. 203-208, abr.-jun., 2011

ISSN 0103-9954

\title{
UMA NOVA ESPÉCIE DE Butia (BECC.) BECC. (ARECACEAE) PARA O RIO GRANDE DO SUL, BRASIL ${ }^{1}$
}

A NEW Butia (BECC.) BECC. SPECIES (ARECACEAE) OF RIO GRANDE DO SUL, BRAZIL

\author{
Kelen Pureza Soares ${ }^{2}$ Solon Jonas Longhi ${ }^{3}$
}

RESUMO

Uma nova espécie de Butia (Becc.) Becc. (Arecaceae): Butia witeckii K. Soares \& S. Longhi, nativa da região central do Rio Grande do Sul - Brasil é descrita e ilustrada. Espécie próxima de Butia yatay (Mart.) Becc. e Butia paraguayensis (Barb. Rodr.) L. H. Bailey, difere destas pelo tamanho e peso do fruto $(3,6-$ 5,6x3,0-4,1 cm com 23,61-43,05 gramas); tamanho, peso e forma do endocarpo/pirênio (2,8-3,5x1,6-2,3 $\mathrm{cm}$ com 3,43-10,11 gramas, providos de três quinas longitudinais e formato quase sempre piramidal no lado dos poros de germinação), e pelo número de pinas (folíolos) de cada lado da raque (43-61 pinas). Difere-se da última espécie também pelo comprimento da parte expandida da bráctea peduncular $(50-85 \mathrm{~cm})$, pelo comprimento da raque da inflorescência $(49-63 \mathrm{~cm})$ e pelo porte $(3,0-6,1$ metros de altura).

Palavras-chave: Arecaceae; Butia; espécie nova; Rio Grande do Sul.

\begin{abstract}
A new Butia (Becc.) Becc. (Arecaceae): Butia witeckii K. Soares \& S. Longhi, native of the central region of Rio Grande do Sul - Brazil is described and illustrated. The species is related to Butia yatay (Mart.) Becc. and Butia paraguayensis (Barb. Rodr.) L.H.Bailey, differing from these two species by the size and weight of the fruit (3.6-5.6x3.0-4.1 cm with 23.61-43.05 grams); size, weight and shape of the endocarp/pyrene (2.8$3.5 \times 1.6-2.3 \mathrm{~cm}$ with $3.43-10.11$ grams, possessing three longitudinal corners and almost always having a pyramidal shape near the germination pore), and by the number of pinnae (leaflets) on each side of rachis (4361 pinnae). Differs from the latter species also by the length of the expanded portion of the peduncular bract $(50-85 \mathrm{~cm})$, by the legth of the inflorescence rachis $(49-63 \mathrm{~cm})$ and by the height of the tree (3.0-6.1 meters).
\end{abstract}

Keywords: Arecaceae; Butia; new specie; Rio Grande do Sul.

\section{INTRODUÇÃO}

Butia (Becc.) Becc. é um gênero composto por 18 espécies sul-americanas, dos quais quatro foram recentemente descritas. Distribui-se pelo Brasil nos estados da Bahia, Goiás, Minas Gerais, Mato Grosso do Sul, São Paulo, Mato Grosso em áreas abertas de cerrado em solo arenoso; pelo sul no Paraná, Santa Catarina, Rio Grande do Sul, onde cresce também junto à vegetação de restinga e campos (HENDERSON et al., 1995; LORENZI et al., 2010; BARBOSA
RODRIGUES, 1903; DRANSFIELD et al., 2008). No Uruguai pode crescer tanto em gleissolos e planossolos alagadiços como em serranias e cerros (CHEBATAROFF, 1971). Também encontrado no Paraguai, Argentina e Bolívia.

No Rio Grande do Sul ocorrem as espécies Butia odorata (Barb. Rodr.) Noblick, Butia catarinensis Noblick \& Lorenzi, Butia lallemantii Deble \& Marchiori, Butia eriospatha (Mart. ex Drude) Becc. e Butia yatay (Mart.) Becc.. A espécie Butia paraguayensis (Barb. Rodr.) L. H. Bailey é de ocorrência duvidosa

1. Parte da Dissertação de Mestrado do primeiro Autor.

2. Engenheiro Florestal, Mestrando do Programa de Pós-graduação em Engenharia Florestal, Centro de Ciências Rurais, Universidade Federal de Santa Maria, Av. Roraima, 1000, CEP 97105-900, Santa Maria (RS). kpsoares@gmail.com

3. Engenheiro Florestal, Dr., Professor Titular do Departamento de Ciência Florestal, Centro de Ciências Rurais, Universidade Federal de Santa Maria, Av. Roraima, 1000, CEP 97105-900, Santa Maria (RS). longhi.solon@gmail.com

Recebido para publicação em 18/07/2010 e aceito em 23/01/2011 
porque até alguns anos atrás Butia lallemantii e Butia paraguayensis eram consideradas a mesma espécie, conhecidas pelo segundo nome e, desde que foram separadas, populações naturais de Butia paraguayensis não foram confirmadas no estado.

Entre a década de 1930 até meados da década de 1970, os butiazais tinham importância econômica no sul do Brasil e Uruguai, pois suas folhas (crina vegetal) eram extraídas e utilizadas na fabricação de colchões e peças de estofaria (BONDAR, 1964). Atualmente algumas espécies são cultivadas e muito valorizadas devido ao sabor dos seus frutos que são consumidos in natura, em forma de geléia, sorvetes e licores. A planta também é utilizada no paisagismo, tanto no Brasil como em países de clima temperado (GEYMONAT e ROCHA, 2009; LORENZI et al., 2010; BONDAR, 1964; DRANSFIELD et al., 2008). Apesar disso, todas as espécies do gênero Butia estão em franco declínio populacional no estado do Rio Grande do Sul e aos poucos vão desaparecendo da paisagem em função da ocupação das áreas de ocorrência com a pecuária extensiva e agricultura (SOARES e WITECK, 2009).

\section{MATERIAL E MÉTODO}

Este estudo faz parte do trabalho de mestrado do primeiro autor, enquadrado no programa de pós-graduação em Engenharia florestal da UFSM, no qual foram analisados, dentre muitos aspectos ecológicos, dados biométricos de 21 exemplares adultos de Butia sp. nov. no habitat natural em Quevedos - RS e comparados com dados de 79 indivíduos da espécie próxima Butia yatay (Mart.) Becc., coletados no habitat natural em Quaraí - RS, bem como, foram feitas consultas a materiais bibliográficos especializados e no Herbário Plantarum (HPL). Dados referentes ao tamanho e peso dos frutos e do endocarpo foram avaliados nas diferentes populações, sendo medidos e pesados frutos de plantas diferentes em ambas as populações. A partir da comparação desses materiais, acompanhamento do desenvolvimento de alguns indivíduos desde a germinação até estágio de plantas jovens no viveiro do Colégio Politécnico da Universidade Federal de Santa Maria e observação de indivíduos cultivados já adultos, chegou-se a conclusão que se trata de uma nova espécie, na qual será descrita e ilustrada.

\section{RESULTADOS E DISCUSÃO}

Butia witeckii K. Soares \& S. Longhi, sp. nov (Figura 1).

Caudex altus, robustus, solitarius, 3,06,1m longus; folia 14-25 contemporanea, in spira, regulariter pinnatisecta; petiolus $27-45 \mathrm{~cm}$ longus, margine spino-serrati; rhachi 100-170 cm longa; foliola opposita in V, utrinque 43-61 per greges 5266x2,3-2,8 cm media; spatha exterior lanceolata, $38 \times 5,3 \mathrm{~cm}$; interior erecta, lignosa, glauca, 118128x7,8-9,7; flores masc. 9-10 $\mathrm{mm}$, sepalis 3 connatis triangularibus et petalis 3 obovatis supra coriaceis; flores fem. 17-20x14-16 mm sessilis, sepalis et petalis convolutis. Drupa 3,6-5,6x3,0$4,1 \mathrm{~cm} ;$ mesocarpio multo fibroso; endocarpio osseo, 2,8-3,5x1,6-2,3 cm, marginibus habet tres longitudinales angulos, faciendo piramidalem formam, in acumine quo sunt porus germinatus. Maiorem fructum et endocarpio generis habet.

Palmeira com 3,0-6,1 m de altura, de estipe solitário coberto pelos remanescentes das bainhas de folhas já caídas, ocasionalmente perdendo este revestimento com a idade, mas deixando visíveis as cicatrizes dispostas de forma helicoidal em torno do estipe, este apresenta 75-110 cm de circunferência. Folhas contemporâneas em número de 14-25; bainha com 37-50 cm de comprimento por $9-13 \mathrm{~cm}$ de largura, e pseudopecíolo (pecíolo mais a parte aparente da bainha) com $27-45 \mathrm{~cm}$ de comprimento e 1,8-2,8 cm de largura, achatado adaxialmente e arredondado abaxialmente, armado com dentes um pouco curvados com 0,5-3,0 cm de comprimento, aumentando de tamanho em direção ao estipe (base), separados entre si por 0,5-4,5 cm e revestido de fibras achatadas quando as folhas são novas, perdendo parte dessas com o tempo; raque das folhas freqüentemente torcida nos indivíduos adultos devido à ação do vento, com 100-170x1,2-2,3 cm, contendo 43-61 pinas (folíolos) distribuídos uniformemente ao longo de cada lado; pinas verde-escuras ou quase glaucas nos indivíduos mais jovens, brilhantes, inseridas em um mesmo plano e formando um "V" com as pinas divergentes do outro lado da raque, as da parte mediana com $52-66 \times 2,3-2,8 \mathrm{~cm}$, todas pinas possuindo mechas de ramento próximas da raque sobre a nervura central na parte inferior e ápice assimétrico. Inflorescências interfoliar, envolvidas por duas brácteas, a mais interna, lenhosa, chamada bráctea peduncular, que protege toda inflorescência até sua abertura (floração), glauca e levemente

Ci. Fl., v. 21, n. 2, abr.-jun., 2011 


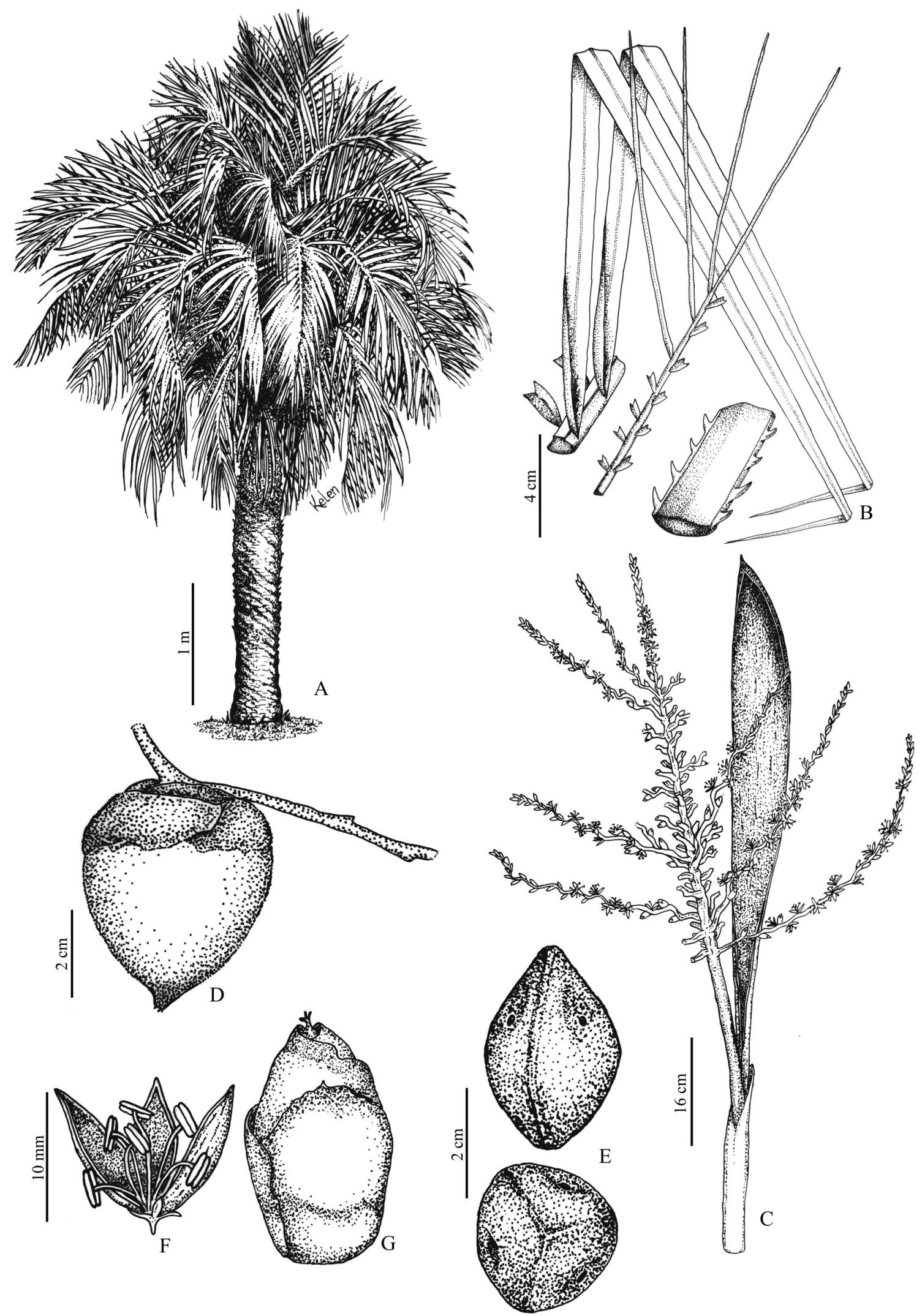

FIGURA 1: Butia witeckii K. Soares \& S. Longhi. A: hábito. B: folha e pseudopecíolo. C: inflorescência. D: fruto. E: endocarpo em vista lateral e superior. F: flor masculina. G: flor feminina. (do tipo). FIGURE 1: Butia witeckii K. Soares \& S. Longhi. A: habit. B: leaf and pseudopetiole C: inflorescense. D: fruit. E: endocarp in lateral and superior. F: male flower. G: female flower. (Type). 
estriada longitudinalmente com 118-128 cm de comprimento total, incluindo o ápice (bico) de até $2 \mathrm{~cm}, 50-85 \mathrm{~cm}$ de comprimento da parte expandida e 7,8-9,7 cm de largura; a bráctea externa, menor, lanceolada, chamada de profilo, fibroso, com 38x5,3 cm fica sempre escondida entre as bainhas das folhas; pedúnculo da inflorescência levemente estriado e coberto por uma fina camada de cera quando novo, com 56-61,5x1,7-2,0 cm; raque da inflorescência verde-clara, ramificada ao nível de primeira ordem, com 49-63 cm de comprimento contendo 66-85 raquilas, as da parte mediana medindo $21,5-38 \mathrm{~cm}$, as da parte basal com $32-50 \mathrm{~cm}$ e da parte terminal com 14-29 cm de comprimento. Flores unissexuais, amarelas, situadas na porção inferior das raquilas em grupo de três, com uma flor central feminina ladeada por duas masculinas, a porção terminal (superior) das raquilas apresenta somente flores masculinas; flores masculinas com 9-10 mm de comprimento, curto pediciladas; pedicelo com cerca de $1 \mathrm{~mm}$ de comprimento; 3 sépalas triangulares, distintas, conatas na base, lobos da sépalas com cerca de $2 \mathrm{~mm}$ de comprimento; pétalas em número de 3 , desiguais, valvadas, estreito obovadas, glabras, agudas, membranácea na parte inferior e coriácea na parte superior (ápice), com 7-8×2-3 mm, possui 6 estames dorsifixos, distintos, com 5-6 mm de comprimento, anteras com 2,5-3,5 mm de comprimento, filetes com 3,0-3,7 mm de comprimento, pistilódio com menos de $1 \mathrm{~mm}$ de comprimento; flores pistiladas mais ou menos ovóides, sésseis, com 17-20x10$13 \mathrm{~mm}$, contendo 3 sépalas e 3 pétalas imbricadas com nervações visíveis apenas no ápice, sépalas com 14-17x14-16 mm, desiguais, mais ou menos triangulares, gineceu com 17-18x9-10 mm, pétalas com 16-19x20-23 mm. Frutos grandes, com 3,6$5,6 \times 3,0-4,1 \mathrm{~cm}$ e com 23,61-43,05 gramas de peso sem incluir o perianto persistente; epicarpo amarelo ou amarelo-esverdeado quando maduro; mesocarpo amarelo claro quando maduro, de sabor agridoce agradável, porém muito fibroso. Endocarpo duro, ósseo, de cor marrom escuro e superfície exterior quase lisa, com 2,7-3,5x1,6-2,3 cm, com 3,4310,11 gramas de peso (pirênio) quando novos, apresenta visivelmente 3 quinas longitudinais bem protuberantes, dando uma forma piramidal nas extremidades do endocarpo (pólos), principalmente no lado dos poros de germinação, os 3 poros de germinação distante $0,7-1,2 \mathrm{~cm}$ do ápice e com menos de 1,6 mm de diâmetro, poros geralmente um pouco depresso no endocarpo; endocarpo com 1-3 sementes estreitas e elípticas quando possui 2-3 lóculos (2-3 sementes) e pouco mais ovais quando possui apenas um lóculo (1 semente); endosperma branco e homogêneo; germinação remota-tubular, com eófilo simples e estreito-lanceolado.

Tipo: Brasil. Rio Grande do Sul: Quevedos, localidade de Quebra Dentes, $2 \mathrm{~km}$ a norte da Usina de Quebra Dentes. S 29²2'07,17' W 5400’45,31. 24/III/2011., fl. fr.; K. P. Soares; C. G. Redin; D. S. Brito. HDCF-6213.

Paratipo: Brasil. Rio Grande do Sul: Quevedos, localidade de Quebra Dentes, em pastagens; $30 \mathrm{~km}$ de terra até a cidade de Toropi. 22/III/2009. fr. H. Lorenzi; R. Pimenta; K. Soares 6651. HPL-11427. Palmeira, ca. 4-6 m de altura, solitária; tronco mostrando a base das folhas caídas; pinas verde-azuladas brilhantes, inflorescências ramificadas ao nível de $1^{\circ}$ ordem, frutos de polpa doce-acidulada, muito fibrosa, amarela.

Distribuição geográfica: Habita a região central do Rio Grande do Sul, nos município de Quevedos, Júlio de Castilhos, São Pedro do Sul, São Martinho da Serra, ao longo do Rio Toropi, em altitudes entre 300-370 metros, crescendo sempre em solos rasos, pedregosos, em locais aberto, com vegetação rala ou associado a algumas espécies arbóreas-arbustivas pioneiras, raramente cresce no interior da mata ciliar ou capões devido a sua exigência por luz. Parte da população cresce junto à maior população da palmeira Trithrinax brasiliensis Mart. do estado; ambas espécies encontram-se bastante ameaçadas pelas atividades agrícolas e pela pecuária.

A palmeira floresce na primavera e o auge da frutificação ocorre no final de março até abril.

O epíteto específico é uma homenagem ao professor Leopoldo Witeck Neto, engenheiro florestal, professor do Colégio Politécnico da Universidade Federal de Santa Maria, que primeiro localizou esta população ainda desconhecida em notas de ocorrência para o gênero Butia, também devido ao seu interesse e colaboração nas pesquisas, bem como por suas atividades conservacionistas.

\section{CONCLUSÃO}

A espécie é próxima de Butia paraguayensis e Butia yatay embora seja facilmente reconhecida em função do tamanho e peso dos seus frutos e tamanho, peso (pirênio) e formato do endocarpo, sendo estes os maiores e mais pesados do gênero Butia (Figura 2), os pólos do endocarpo dos frutos apresentam formato piramidal, principalmente

Ci. Fl., v. 21, n. 2, abr.-jun., 2011 
no lado dos poros de germinação, devido as 3 quinas longitudinais; o mesocarpo dos frutos é extremamente fibroso comparado com qualquer outra espécie do gênero; este padrão é mantido mesmo em indivíduos cultivados fora do habitat natural. $\mathrm{O}$ auge da frutificação ocorre mais tarde que a maioria das espécies de Butia, em marçoabril, provavelmente por demandar mais tempo para formação dos grandes frutos. Diferente das outras espécies, as sementes de Butia witeckii raramente é predada por insetos, porém é bastante procurada pelo serelepe (Sciurus ingrami Thomas, 1901). O porte médio dos indivíduos adultos é mais alto e possui um comprimento da raque da inflorescência maior que a espécie Butia paraguayensis. Apresenta um número menor de pinas (folíolos) em cada lado da raque e em geral pinas mais largas que Butia yatay, além disso, o epicarpo dos frutos não apresenta cor vermelha como geralmente acontece em Butia yatay. $\mathrm{O}$ desenvolvimento das plântulas em viveiro tem mostrado ser mais lenta que as da espécie Butia yatay.

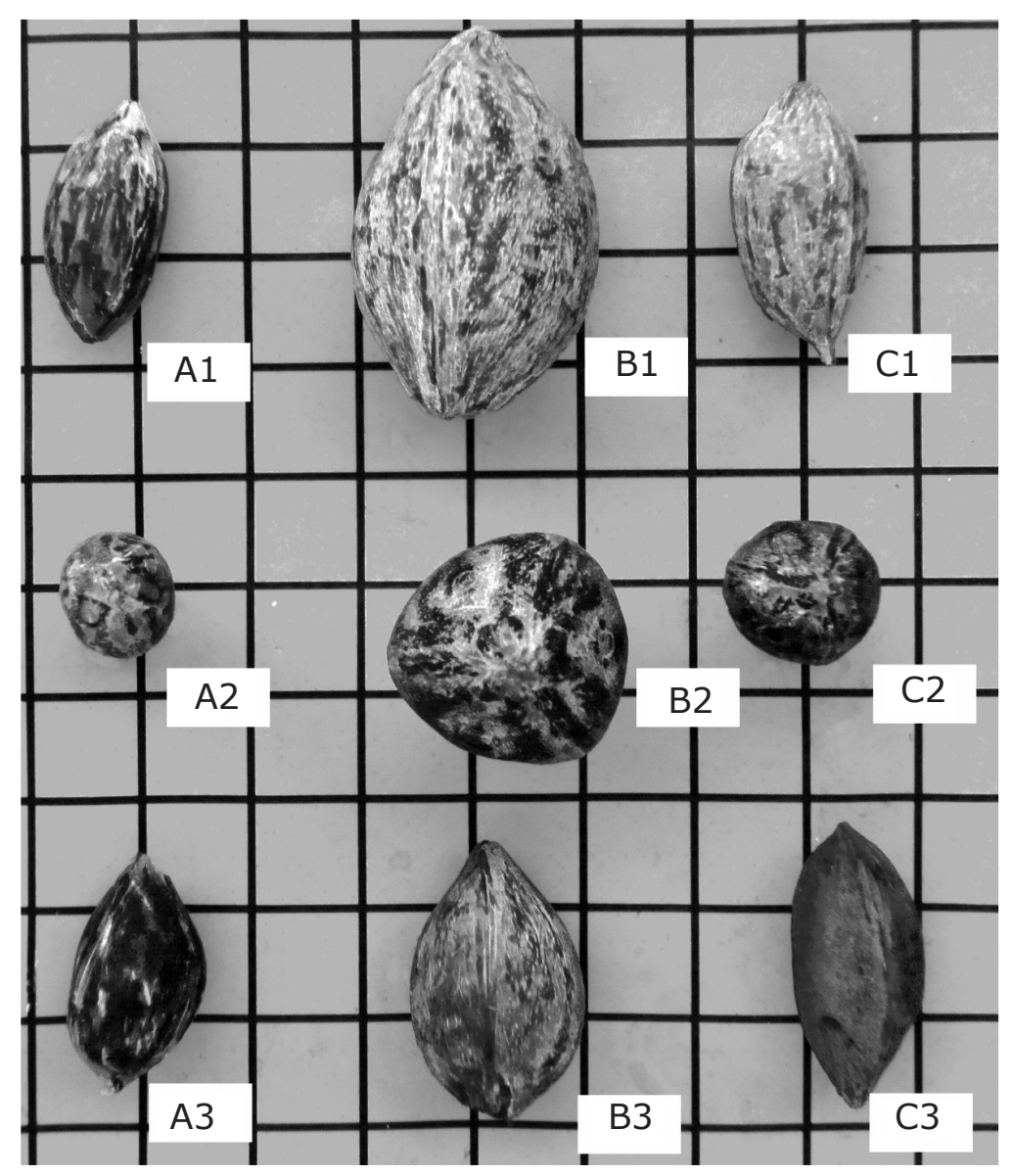

FIGURA 2: Endocarpos. A1: Butia paraguayensis (Barb. Rodr.) L. H. Bailey vista lateral; A2: Butia paraguayensis (Barb. Rodr.) L. H. Bailey vista superior; A3: Butia paraguayensis (Barb. Rodr.) L. H. Bailey endocarpo mais largo encontrado; B1: Butia witeckii K. Soares \& S. Longhi vista lateral; B2: Butia witeckii K. Soares \& S. Longhi vista superior; B3: Butia witeckii K. Soares \& S. Longhi um dos menores férteis encontrados; C1: Butia yatay (Mart.) Becc. vista lateral; C2: Butia yatay (Mart.) Becc. vista superior; C3: Butia yatay (Mart.) Becc. um dos maiores encontrados.

FIGURE 2: Endocarp. A1: Butia paraguayensis (Barb. Rodr.) L. H. Bailey lateral view; A2: Butia paraguayensis (Barb. Rodr.) L. H. Bailey top view; A3: Butia paraguayensis (Barb. Rodr.) L. H. Bailey wider endocarp found; B1: Butia witeckii K. Soares \& S. Longhi side view; B2: Butia witeckii K. Soares \& S. Longhi top view; B3: Butia witeckii K. Soares \& S. Longhi one of the smallest fertile found; C1: Butia yatay (Mart.) Becc. lateral view; C2: Butia yatay (Mart.) Becc. top view; C3: Butia yatay (Mart.) Becc. one of the biggest found. 
Chave dicotômica para identificação de espécies do gênero Butia ocorrentes no estado do Rio Grande do Sul (incluindo Butia paraguayensis de ocorrência natural duvidosa)

1. Flores femininas com até $9 \mathrm{~mm}$ de comprimento; endocarpo dos frutos arredondados ou ligeiramente ovais

2. Bráctea peduncular revestida por denso tomento lanuginoso de coloração castanha ..... Butia eriospatha

2. Bráctea peduncular lisa, glauca, sem tomento lanuginoso

3. Planta com caule até 2 metros de altura e $15-43 \mathrm{~cm}$ de diâmetro; bráctea peduncular da inflorescência mais larga acima da porção intermediária e terminando abruptamente em um bico curto; frutos mais compridos que largos, estreito-oblongo

Butia catarinensis

3. Planta com caule até 9 metros de altura e $32-58 \mathrm{~cm}$ de diâmetro; bráctea peduncular da inflorescência mais largas próximo da porção média da parte expandida e afinando gradualmente em direção ao ápice; frutos freqüentemente mais largos que compridos

Butia odorata

1. Flores femininas com mais de $10 \mathrm{~mm}$ de comprimento; endocarpo alongado, nunca arredondado

2. Plantas com capacidade emitir perfilhos (caule cespitoso).

Butia lallemantii

2. Planta sem capacidade de perfilhar (caule único)

3. Plantas adultas acaulescentes ou com caule de até 3 metros; parte expandida da bráctea peduncular entre 40-60 cm de comprimento; raque da inflorescência com 13-30 cm de comprimento.

Butia paraguayensis

3. Plantas adultas com 3-9 (-15) metros de altura; parte expandida da bráctea peduncular entre $55-85 \mathrm{~cm}$ de comprimento; raque da inflorescência com $47-72 \mathrm{~cm}$ de comprimento

4. Folhas com 43-61 pinas de cada lado da raque; pinas da parte mediana da raque com 40-65 x 2,3$2,8 \mathrm{~cm}$; frutos maduros com 3,6-5,6 x 3,0-4,1 cm, pesando entre 23,61-43,05 gramas; endocarpo de frutos novos com 2,8-3,5 x 1,6-2,3 cm e pesando entre 3,61-10,11 gramas (pirênio) com 3 quinas longitudinais visíveis, conferindo uma forma quase piramidal no lado onde ficam os poros de germinação

Butia witeckii

4. Folhas com (57-) 63-78 pinas de cada lado da raque; pinas da parte mediana da raque com (58-) 65-77 x 2,0-2,4 cm; frutos maduros com 3,0-4,1 x 1,5-2,8 cm, pesando entre 8,9115,39 gramas; endocarpo de frutos novos com 1,8-3,0 x 1,0-1,4 cm e pesando entre 1,42-2,96 gramas (pirênio) sem quinas longitudinais e com forma de cone no lado onde ficam os poros de germinação

Butia yatay

\section{AGRADECIMENTOS}

Agradecemos à mestranda Cristina Gouvea Redin e ao estudante de engenharia florestal Douglas Senger de Brito pela grande ajuda durante as coletas de campo. À professora Leila Maraschin pela diagnose em latim.

\section{REFERÊNCIAS BIBLIOGRÁFICAS} BARBOSA RODRIGUES, J. Sertum Palmarum
Brasiliensium. Fac-símile de: Bruxelles: Imprimerie Typographique Veuve Monnom, 1903. BONDAR, G. Palmeiras do Brasil. São Paulo - SP: Secretaria da Agricultura do Estado de São Paulo - Instituto de Botânica; Editora Gráfica Irmãos Andrioli S/A., 1964.

CHEBATAROFF, J. Condiciones Ecológicas que Influyen em La Distribuición de lãs Palmeras Del Uruguay. Montevideo, Uruguay: Facultad de
Humanidades y Ciências, 1971. 24p. Trabajos de investigación y de revisión $\mathrm{n}^{\circ} 4$.

DRANSFIELD, J. et al. Genera Palmarum: Evolution and Classification of Palms. Kew: Kew Publishing, Royal Botanic Gardens, 2008.

GEYMONAT, G.; ROCHA, N. Butia: Ecossistema único em el mundo. Castillos, Rocha, Uruguay: Casa Ambiental, 2009.

HENDERSON, A.; GALEANO, G.; BERNAL, R. Field Guide to the Palms of the Americas. New Jersey: Princeton University, 1995.

LORENZI, H. et al. Flora Brasileira: Arecaceae (Palmeiras). Nova Odessa-SP: Instituto Plantarum, 2010.

SOARES, K.; WITECK, L. Ocorrência de Butia capitata e outras espécies do gênero Butia na região central do Rio Grande do Sul, Brasil. In: GEYMONAT, G.; ROCHA, N. (Eds.). Butia: Ecossistema único em el mundo. Castillos, Rocha, Uruguay: Casa Ambiental, 2009. p.37-41.

Ci. Fl., v. 21, n. 2, abr.-jun., 2011 